\title{
Article \\ Structural Damage Identification Using a Modified Directional Bat Algorithm
}

\author{
Yonghui Su, Lijun Liu* and Ying Lei \\ Department of Civil Engineering, Xiamen University, Xiamen 361005, China; suyh97@163.com (Y.S.); \\ ylei@xmu.edu.cn (Y.L.) \\ * Correspondence: liulj214@xmu.edu.cn
}

Citation: Su, Y.; Liu, L.; Lei, Y. Structural Damage Identification Using a Modified Directional Bat Algorithm. Appl. Sci. 2021, 11, 6507. https://doi.org/10.3390/ app11146507

Academic Editor: Mohammad Noori

Received: 21 June 2021

Accepted: 12 July 2021

Published: 15 July 2021

Publisher's Note: MDPI stays neutral with regard to jurisdictional claims in published maps and institutional affiliations.

Copyright: (c) 2021 by the authors. Licensee MDPI, Basel, Switzerland. This article is an open access article distributed under the terms and conditions of the Creative Commons Attribution (CC BY) license (https:// creativecommons.org/licenses/by/ $4.0 /)$.

\begin{abstract}
Bat algorithm (BA) has been widely used to solve optimization problems in different fields. However, there are still some shortcomings of standard BA, such as premature convergence and lack of diversity. To solve this problem, a modified directional bat algorithm (MDBA) is proposed in this paper. Based on the directional bat algorithm (DBA), the individual optimal updating mechanism is employed to update a bat's position by using its own optimal solution. Then, an elimination strategy is introduced to increase the diversity of the population, in which individuals with poor fitness values are eliminated, and new individuals are randomly generated. The proposed algorithm is applied to the structural damage identification and to an objective function composed of the actual modal information and the calculated modal information. Finally, the proposed MDBA is used to solve the damage detection of a beam-type bridge and a truss-type bridge, and the results are compared with those of other swarm intelligence algorithms and other variants of BA. The results show that in the case of the same small population number and few iterations, MDBA has more accurate identification and better convergence than other algorithms. Moreover, the study on anti-noise performance of the MDBA shows that the maximum relative error is only $5.64 \%$ at $5 \%$ noise level in the beam-type bridge, and $6.53 \%$ at $3 \%$ noise in the truss-type bridge, which shows good robustness.
\end{abstract}

Keywords: directional bat algorithm; structural damage identification; constrained optimization problem; modal parameters; objective function

\section{Introduction}

Due to the diversity of loads and the complexity of working environment, civil engineering structures often suffer various damages during service, such as concrete cracking, steel yield and so on. Structural damage could cause the change of structural physical characteristics (stiffness, mass, damping, etc.) and cause the variation of modal characteristics (natural frequency, modal shape, etc.). These damages also affect the safety of the structure to a certain extent, so it is very important to find the damage of the structure in time. Goyal et al. [1] gave an introduction to various methods for structural damage identification using vibration response data and vibration characteristics, such as acceleration response, natural frequency, vibration mode and flexibility. However, these measurement data are often affected by environmental factors, experimental errors and other uncertain factors, which lead to their failure to truly reflect the status of the structure. Therefore, how to use the structural health identification technology to evaluate the reliability, integrity and safety of the structure is particularly important [2].

In recent years, with the development of the computer, the combination of the swarm intelligence optimization algorithm (SIOA) and structural damage identification has become a popular direction in the field of structural health monitoring (SHM). SIOA transforms a complex structural damage identification into a constrained optimization problem. There are many kinds of SIOA, such as the classical genetic algorithm (GA) [3], particle swarm optimization (PSO) [4], differential evolution algorithm (DE) [5,6], simulated 
annealing algorithm (SA) [7], ant colony optimization (ACO) [8] and so on. In recent years, many new algorithms have emerged, such as the whale optimization algorithm (WOA) [9], bat algorithm (BA) [10], ant lion optimizer (ALO) [11], shuffled frog leaping algorithm (SFLA) [12], cuckoo algorithm (CS) [13], grey wolf optimizer (GWO) [14], fruit fly optimization algorithm (FOA) [15,16], backtracking search algorithm (BSA) $[17,18]$ and so on. Swarm intelligence algorithms have been applied to a variety of problems. For example, SFLA algorithms have been used for the traveling salesman problem and maximum power point tracking problems and so on $[19,20]$. Each algorithm has its own characteristics and shortcomings. For example, the GA algorithm is easy to implement and has a fast convergence speed, but it lacks global search ability. The PSO algorithm has simple structure and fast convergence speed, and it is easy to fall into a local optimal solution. The ACO algorithm has strong global search ability, is easy to combine with other evolutionary algorithms, has low convergence speed, is easy to fall into a local optimal solution and so on. Many SIOAs have been employed in structural damage identification. Chen et al. [21] proposed a hybrid ant lion optimizer. Based on the damage identification objective function of modal parameters, the numerical simulation of a two-story rigid frame structure and plane truss structure was carried out, and the effectiveness and feasibility of the method were verified. Du et al. [22] used the Jaya algorithm to determine the damage location and damage degree of truss and frame structures based on multi damage location assurance criteria and modal flexibility changes. Roumaissa et al. [23] compared the accuracy of the GA algorithm and Bat algorithm through the objective function composed of a measured frequency response function and calculated frequency response function. The substructure, strain energy and SIOA were used to identify the damage of a large spatial structure $[24,25]$, in which the substructure was used to divide the large spatial structure, the strain energy was used to locate the structural damage, and SIOA was used to quantify the structural damage. It is worth noting that in addition to the above modal parameters, the objective function can also be constructed by using the structural response data. Ding et al. [26] proposed a Gauss tree seed algorithm to identify the structural damage of simply supported beam and truss structures based on the objective function composed of the absolute error of acceleration response. Zhu et al. [27] proposed a damage identification method of a bird mating optimizer, which used the bird mating optimizer to identify the structural damage based on the objective function composed of frequency and acceleration. Although most of the above-mentioned SIOAs have achieved good success in solving constrained optimization problems of structural damage identification, there are still problems like large population and too many iterations, which lead to complex calculation and long calculation time, especially when using structural response data as the error objective function.

The bat algorithm (BA) is a random search algorithm proposed by Yang [28], which simulates bats in nature using sonar to detect prey and avoid obstacles. It consists of three parts: global search, local search and parameter update. BA has been widely used in different fields because of its few parameters, fast convergence and feasibility [29]. However, the standard BA is a kind of searching mechanism close to the current optimal solution, which makes it easy to fall into the problem of local optimal solution, resulting in it being unable to find the optimal solution of the objective function. To solve this limitation, many researchers have improved the standard BA by enhancing the search ability and population diversity. He et al. [30] modified the global search formula of the standard BA through Levy flight to enhance the search ability of the algorithm, and they used it to solve the logistics planning problem. Gan et al. [31] combined BA with an iterative local search algorithm to enhance the diversity of population, add stochastic inertia weight to adjust search step length and balance search mode by linear updating pulse rate and loudness. Liu et al. [32] combined the improved time factor, Gaussian mutation factor and Cauchy mutation factor with BA to improve the performance of BA in the optimization problem, and they applied them to the problem of low-velocity impact localization. Cui et al. [33] proposed an adaptive BA to optimize the observation matrix, and they designed a dynamic 
adjustment strategy of the optimal radius to improve its global convergence ability. In addition to the above bat variants, there are many other variants, such as Island Bat [34], directional Bat algorithm (DBA) [35], Hybrid Bat algorithm [36], Enhanced Bat [37], Novel chaotic Bat [38], Chaotic bat swarm optimization [39] and so on. It can be seen that many improved BAs have made some progress in different fields, but the above methods are not well applied to the problem of structural damage identification. For the problem of structural damage identification, the BA needs to be improved to find out the location and degree of structural damage accurately under the condition of small population and few iterations.

In this paper, a modified directional bat algorithm (MDBA) has been proposed, which combines the individual optimal updating mechanism, elimination strategy and DBA, and it is applied to the optimization problem of structural damage identification. The beam-type bridge model and truss-type bridge model are used for numerical simulation. The structural damage is expressed by linear reduction of element stiffness, and the error objective function is constructed by structural modal information. Under the condition of low population and few iterations, the MDBA is compared with the differential evolution algorithm (DE), particle swarm optimization algorithm (PSO), shuffled frog leaping algorithm (SFLA) and several variants of BA. Furthermore, the robustness of the algorithm is tested by using different degrees of noise. The results show that the MDBA can be used as an efficient application tool to solve the problem of structural damage identification.

The remainder of this paper is as follows. The second section introduces the establishment of the objective function. The third section introduces the modified directional bat algorithm in detail. In the fourth section, a beam-type bridge and a truss-type bridge are used to compare the effects of different optimization algorithms and bat variants in damage identification. Finally, the research results are analyzed, and the conclusion is drawn.

\section{Theoretical Background}

In the problem of structural damage identification based on the optimization algorithm, by adjusting the parameters of the finite element model, the response data of the finite element model is close to that of the actual model, so that the structural parameters of the actual model can be obtained. The key is to reduce the error between the measured data and the calculated data as much as possible. In this paper, the modal information data are used to construct the objective function, and the specific process is shown in Figure 1.

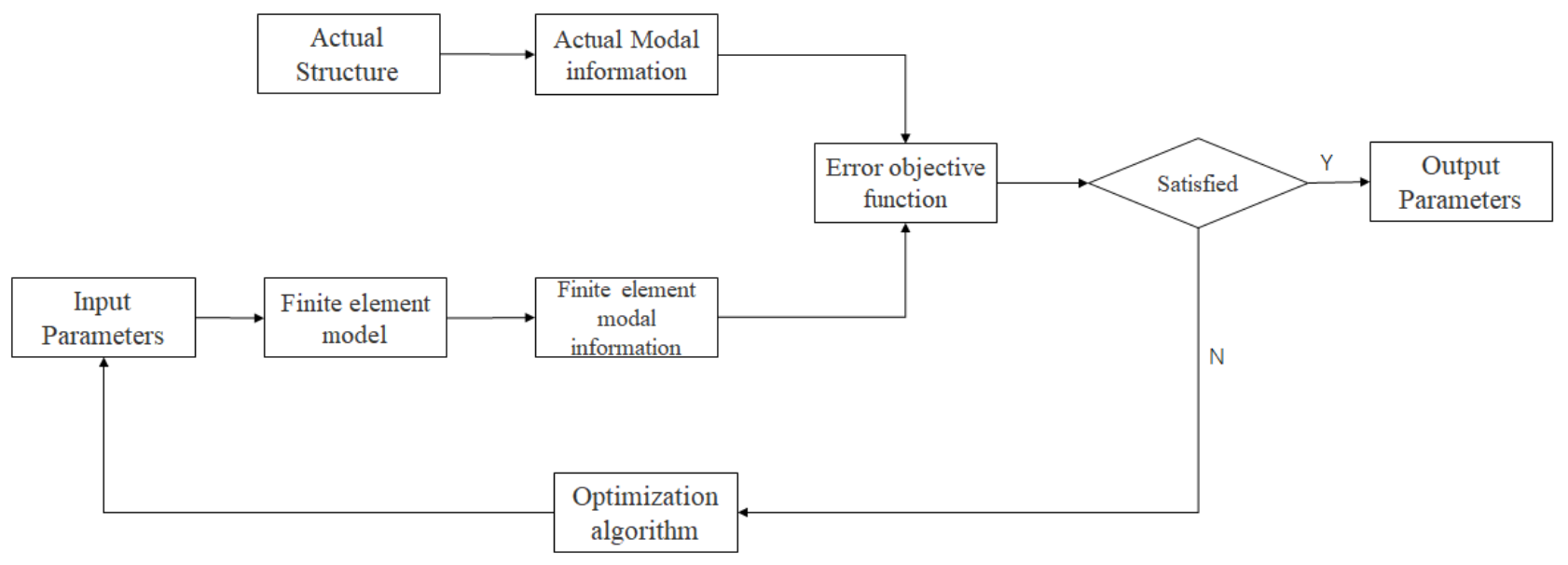

Figure 1. The damage identification problem is transformed into the optimization problem.

It is well known that the eigenvalue equations of the structural finite element model are as follows:

$$
\left(K-\omega_{i}^{2} M\right) \phi_{i}=0
$$


where $K$ is the global stiffness matrix, $M$ is the global mass matrix, $\omega_{i}$ is the $i$-th natural frequency and $\phi_{i}$ is the corresponding mode shape.

In this paper, the linear reduction of structural stiffness is used to simulate the structural damage. The damage of each structural element can be expressed by the damage factor of each element; thus, the stiffness matrix of the element is expressed as follows:

$$
K^{d}=\sum_{i=1}^{N e l}\left(1-\alpha_{i}\right) k_{i}^{e}
$$

where $k_{i}^{e}$ is the stiffness of the $i$-th element in the healthy structure, $N e l$ is the total number of structural elements and $K^{d}$ is the overall structural stiffness of the structure to be identified. $\alpha_{i}$ is the damage degree of $i$-th element of the structure to be identified. When $\alpha_{i}=0$, it means that the structure is healthy; $\alpha_{i}=1$ means that the structure is completely damaged.

In the structural damage identification, the objective function can reflect the difference between real measurement and simulation. Structural modal properties are often used as an index of objective function. The most common modal indicators are the relative error of natural frequency and the modal assurance criterion. Usually, the objective function only uses the first several order modal information of the structure for calculation. In this study, the natural frequency, mode shape and flexibility matrix of the structure are used to form the objective function. The error function of frequency is expressed as follows:

$$
\operatorname{MDLAC} C_{i}=\frac{\left|\omega_{i}^{m}-\omega_{i}^{c}\right|}{\omega_{i}^{m}}
$$

where $\omega_{i}^{m}$ and $\omega_{i}^{c}$ are the $i$-th order measured natural frequency and calculated natural frequency, respectively.

The modal assurance criterion is used to check the correlation between modes. When the measured mode shape is closer to the calculated mode shape, the modal confidence is closer to 1 , otherwise it is 0 . The expression of the modal assurance criterion is as follows:

$$
M A C_{i}=\frac{\left|\phi_{i}^{m} \bullet \phi_{i}^{c}\right|^{2}}{\left|\phi_{i}^{m} \bullet \phi_{i}^{m}\right|\left|\phi_{i}^{c} \bullet \phi_{i}^{c}\right|}
$$

where $\phi_{i}^{m}$ and $\phi_{i}^{c}$ are the $i$-th measured mode shape and calculated mode shape, respectively.

It is well known that the stiffness matrix and the flexibility matrix are reciprocal matrices. The decrease of structural stiffness will increase the corresponding flexibility, and the flexibility matrix is more sensitive to damage [40-42]. The expression of the flexibility assurance criterion is as follows:

$$
M A C F_{i}=\frac{\left|F_{i}^{m} \bullet F_{i}^{c}\right|^{2}}{\left|F_{i}^{m} \bullet F_{i}^{m}\right|\left|F_{i}^{c} \bullet F_{i}^{c}\right|}
$$

where $F_{i}^{m}$ and $F_{i}^{c}$ are the $i$-th order measurement flexibility vector and calculation flexibility vector, respectively. The flexibility matrix can be obtained by the following formula:

$$
[F]=\sum_{i=1}^{s} \frac{1}{\omega_{i}^{2}}\left\{\phi_{i}\right\}\left\{\phi_{i}\right\}^{T}
$$

The objective function is defined as follows:

$$
\text { Function }=\sum_{i=1}^{s}\left[\Delta_{1}\left(1-M A C_{i}\right)+\Delta_{2} M D L A C_{i}+\Delta_{3}\left(1-M A C F_{i}\right)\right]
$$

where $s$ is the order obtained. In order to distinguish the sensitivity of natural frequency, mode shape and flexibility matrix in structural damage identification, the objective function is weighted [21,43]. The weight coefficient of the objective function is determined to be $\Delta_{1}=0.82, \Delta_{2}=0.13, \Delta_{3}=0.05$ and $\Delta_{1}+\Delta_{2}+\Delta_{3}=1$.

Considering the influence of uncertain factors on modal parameters, random noise is added. The expression is as follows:

$$
x_{\text {noise }}=x\left(1+\eta R_{n}\right)
$$


where $x_{\text {noise }}$ and $x$ are the modal parameters with and without noise, respectively; $\eta$ is the noise level; $R_{n}$ is a random matrix, and each number in the matrix is a random number of $[0,1]$.

\section{Modified Directional Bat Algorithm (MDBA)}

Standard BA is a swarm intelligence heuristic search algorithm proposed by Yang [28], which is a random search algorithm that simulates bats in nature using sonar to detect prey and avoid obstacles. It is mainly composed of exploration and exploitation. Exploration is a rough search for undeveloped areas, while exploitation is a fine search for developed areas. Standard BA is a kind of algorithm close to the optimal solution. If the initial position distribution is too concentrated, it would easily lead to the algorithm falling into the local optimum. To overcome this deficiency, Chakri et al. [35] proposed the DBA, in which the directional echolocation and three other modifications were embedded into the BA to increase its exploitation and exploration capabilities. However, DBA still has the problems of a large population and too many iterations, and it is not well applied in structural damage identification under the condition of a small population and few iterations. Thus, a modified DBA (MDBA) is proposed here to solve the above limitation, in which the individual optimal renewal mechanism and elimination strategy are employed to enhance the search algorithm performance.

Inspired by the position update mechanism of PSO and combined with the individual historical optimum position, the exploration formula of DBA has been improved in this study. Individual historical optimum position refers to one of the best positions of each particle in the iteration process. As shown in Figure 2, the current optimal solution is in a local optimal situation. Without combining the individual optimal solution, other bats will be close to the current optimal solution, resulting in the algorithm being unable to identify the optimal solution of the objective function. When the individual optimum is added, the other bats are subject to the traction of the global optimum bat position on the one hand, and the traction of their own optimum position on the other hand. By adjusting the parameters of the step size, the possibility of jumping out of the local optimum is increased. Moreover, if the bat is in the individual optimal position, the value of the individual optimal term is 0 . Therefore, the exploration formula is combined with the individual optimal solution, and the expression formula is as follows:

$$
\begin{gathered}
f_{i}=f_{\min }+\left(f_{\max }-f_{\min }\right) \text { rand } \\
\begin{cases}x_{i}^{t+1}=x_{i}^{t}+\left(x_{\text {best }}-x_{i}^{t}\right) f_{1}+\left(x_{k}^{t}-x_{i}^{t}\right) f_{2}+\left(x_{i}^{*}-x_{i}^{t}\right) f_{3} & f\left(F\left(x_{v}^{t}\right)<F\left(x_{i}^{t}\right)\right) \\
x_{i}^{t+1}=x_{i}^{t}+\left(x_{\text {best }}-x_{i}^{t}\right) f_{1}+\left(x_{i}^{*}-x_{i}^{t}\right) f_{3} & \text { else }\end{cases}
\end{gathered}
$$

where $x_{i}^{t}$ is the current position of the $i$ th bat $(i=1, \ldots, m), m$ is the number of the bat population, $x_{i}^{t+1}$ is the updated position of the $i$ th bat, $x_{k}^{t}$ is the randomly selected bat position $(k \neq i), x_{i}^{*}$ is the individual optimum solution for the $i$ th bat in the search process, $f_{i}$ is the frequency of the $i$ th bat and $F(\bullet)$ is the function value of substituting the bat into the objective function. Rand is a random number generated by a uniform distribution in the range of $[0,1]$.

To increase the diversity of the population, broaden the search range and avoid the possibility of falling into the local optimum, the elimination strategy is used in this paper. It is used to rank bats in a population according to their fitness during the iteration, and the lower-ranked bats are eliminated, and a corresponding number of new bats are added based on their current optimal location. The new bats randomly inherit a certain number of coordinates from the optimum bat, while the remaining coordinates are randomly generated within the search range. The elimination strategy can perturb the bat population and give the BA a chance to jump out of the local optimum. This strategy improves the quality of the bat population well, eliminates bats with poor fitness values, generates new bats based on the optimal bats and increases the diversity of the bat population in the algorithm. 


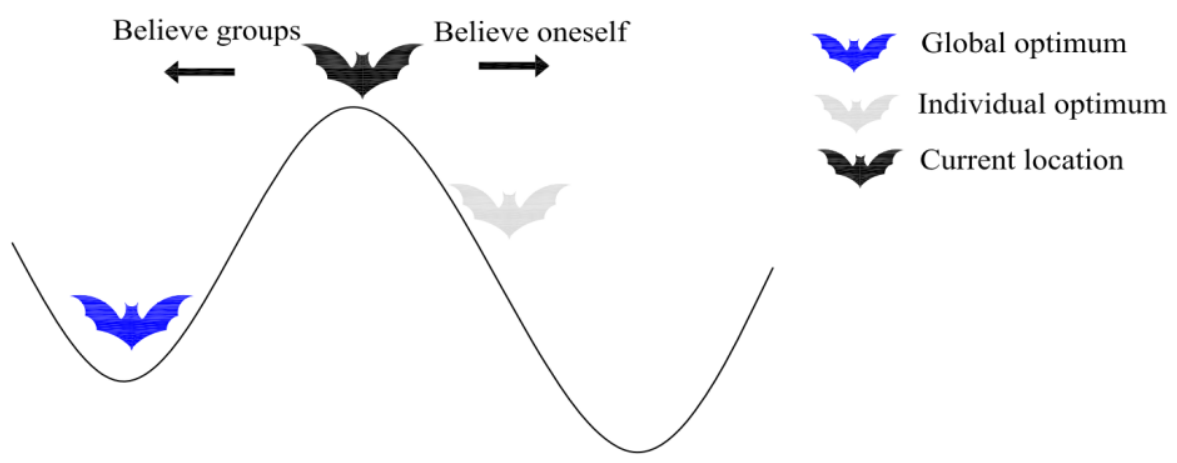

Figure 2. The influence of the individual optimum.

The detailed procedures of MDBA are as follows:

1. Give different initial values of parameters, bat population $m$, iteration number $N$, initial value of loudness $A_{0}$, final value of loudness $A_{\infty}$, initial value of pulse rate $r_{0}$, final value of pulse rate $r_{\infty}$, maximum frequency $f_{\max }$, minimum frequency $f_{\min }$ upper limit of variable $U b_{i}$, and lower limit of variable $L b_{i}$.

2. Give each bat a random initial position $x_{i}(i=1,2,3, \ldots \ldots, m)$, and select the optimal solution from the initial bat $x_{\text {best }}$.

3. Update bat frequency and location according to Equations (9) and (10).

4. When the condition ( rand $>r_{i}$ ) is satisfied, each bat uses the local search Equation (11) to generate a new location locally.

$$
x_{i}^{t+1}=x_{i}^{t}+A^{t} \varepsilon w_{i}^{t}
$$

where $A^{t}$ is the average loudness of all bats, and $\varepsilon \in[-1,1]$ is a random vector. $w_{i}^{t}$ is a parameter that regulates the search scale during iteration. It starts with a larger value (about a quarter of the search domain) and then decreases to $1 \%$ of the initial value. The updating formula of $w_{i}^{t}$ is as follows:

$$
w_{i}^{t}=\left(\frac{w_{i 0}-w_{i \infty}}{1-t_{\max }}\right)\left(t-t_{\max }\right)+w_{i \infty}
$$

5. When the condition (rand $<A_{i} \& F\left(x_{i}^{t+1}\right)<F\left(x_{i}^{t}\right)$ is met, update parameters $r_{i}$ and $A_{i}$ according to the following rules:

$$
\begin{aligned}
r^{t} & =\left(\frac{r_{0}-r_{\infty}}{1-t_{\max }}\right)\left(t-t_{\max }\right)+r_{\infty} \\
A^{t} & =\left(\frac{A_{0}-A_{\infty}}{1-t_{\max }}\right)\left(t-t_{\max }\right)+A_{\infty}
\end{aligned}
$$

6. Update the optimal solution.

7. If the elimination strategy is satisfied, population adjustment is carried out, otherwise perform the next step.

8. Judge if the maximum number of iterations is reached, and if not, the next will be performed.

9. At the end of the iteration, the optimal solution that minimizes the value of the objective function is the output.

Figure 3 is a flow chart of MDBA. 


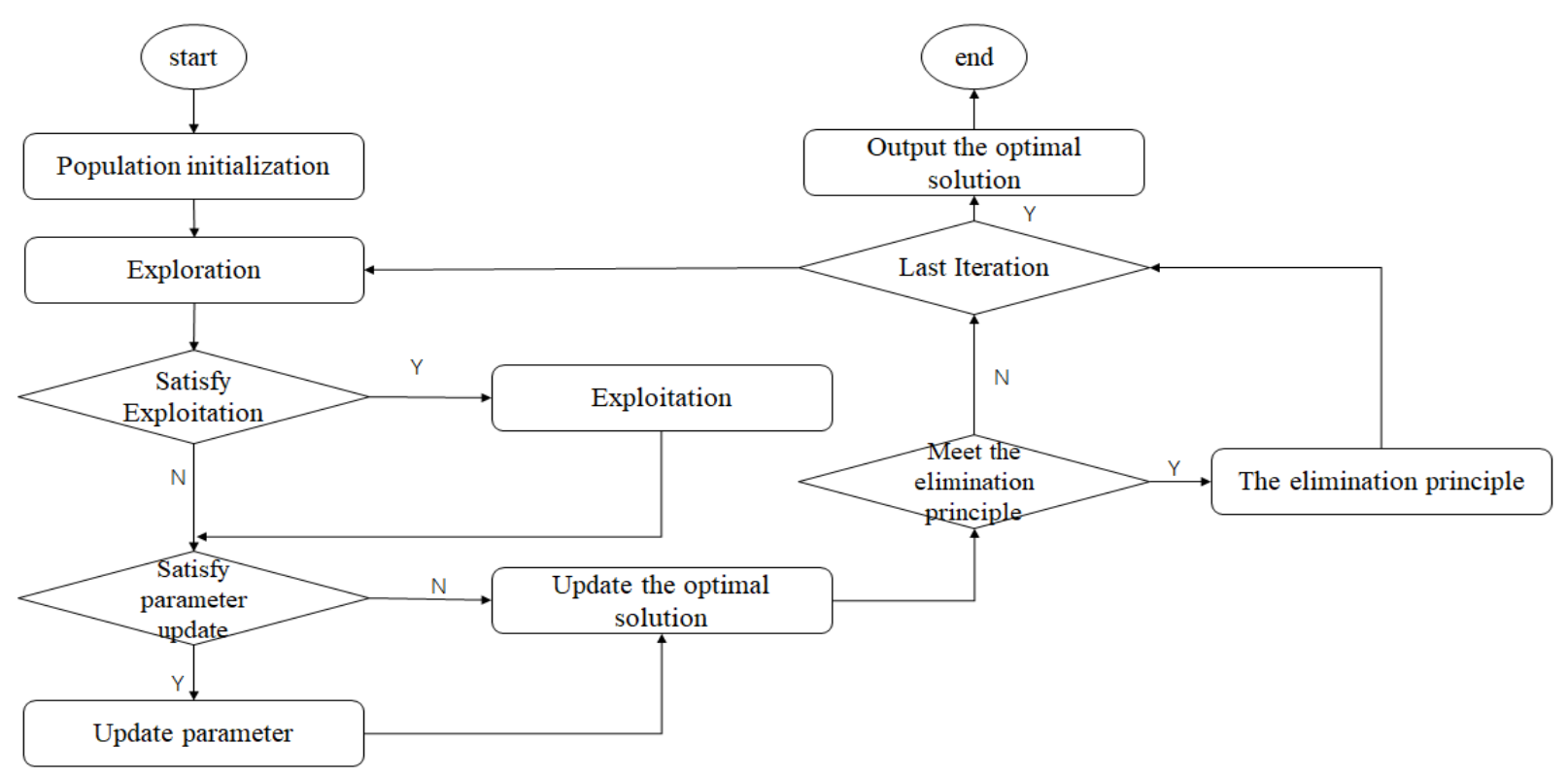

Figure 3. The flow chart of MDBA.

\section{Numerical Simulation}

A beam-type bridge model and a truss-type bridge model have been used as numerical examples to verify the convergence, accuracy and robustness of the proposed MDBA under the condition of low population and few iterations. The MDBA is compared with PSO, DE, SFLA and several variants of BA. All the algorithms have the same population number and iterations times in the different simulations, and the population number is set to 40 , and the number of iterations is set to 100. The parameters of these algorithms are set as follows $[12,19,35,44]$, MDBA: The parameters' settings are $r_{0}=0.1, r_{\infty}=0.7, A_{0}=0.9$, $A_{\infty}=0.6, f_{\min }=0$ and $f_{\max }=2$. SFLA: The parameters' settings are $m=5, L=25$ and $S_{\max }=100 \%$. PSO: The parameters' settings are $c 1=1.49$ and $c 2=1.49$. DE: The parameters' settings are $C R=\operatorname{rand}[0.2,0.9]$ and $F=\operatorname{rand}[0.4,1]$. DBA: The parameters' settings are $r_{0}=0.1, r_{\infty}=0.7, A_{0}=0.9, A_{\infty}=0.6, f_{\min }=0$ and $f_{\max }=2$. LBA: The parameters' settings are $r=0.1, A=0.9, f_{\min }=0, f_{\max }=2, \alpha=0.9, \gamma=0.9$ and $\beta=1.7$. TMBA: The parameters' settings are $r=0.1, A=0.9, f_{\min }=0, f_{\max }=2, \alpha=0.9$ and $\gamma=0.9$.

\subsection{Beam-Type Bridge Model}

The beam-type bridge model is shown in Figure 4, with a total of 10 elements and 11 nodes. The moment of inertia of the beam is $I=7.617 \times 10^{-7} \mathrm{~m}^{4}$, the young's modulus of elasticity is $E=2.1 \times 10^{11} \mathrm{~Pa}$, the mass density is $\rho=7850 \mathrm{kgm}^{-3}$ and the cross-sectional area is $A=1.164 \times 10^{-3} \mathrm{~m}^{2}$. The first five natural frequencies and the corresponding modes of vertical degrees of freedom are used to identify the structural damage.

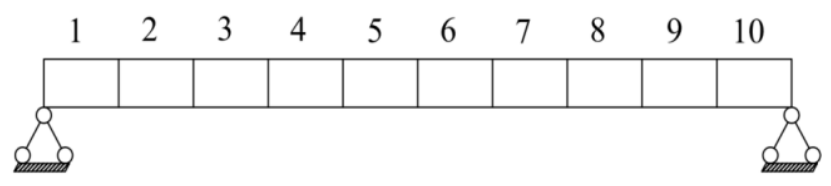

Figure 4. Beam-type bridge model.

The numerical simulation process is completed by matlab2020b software. The damage of the beam-type bridge model is shown in Figure 5. The stiffness of the second element and the seventh element are reduced by $20 \%$ and $25 \%$, respectively. 


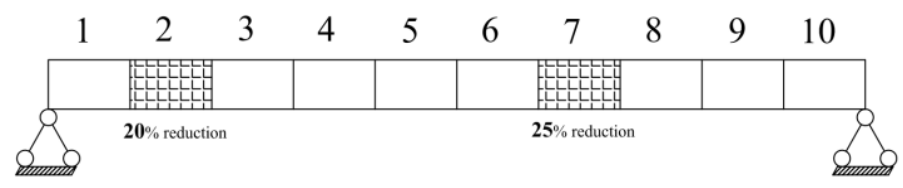

Figure 5. Case of damage beam-type bridge model.

The stiffness is identified by MDBA, PSO, DE and SFLA under the same population number and iteration times. The convergence graph and identification result without noise of different optimization algorithms are shown in Figure 6 and Table 1. It is clear that all the algorithms could identify the damage location. From the point of view of identification accuracy, MDBA had the highest identification accuracy, and its maximum relative error was only $0.05 \%$. The maximum relative error of other algorithms was $1.36 \%$ (SFLA), $20 \%$ (PSO) and $10.95 \%$ (DE), respectively. The accuracy of SFLA was better than that of PSO and DE, but SFLA and PSO had small misjudgments in the undamaged area. As can be seen from Figure 6a, the MDBA algorithm had the fastest convergence speed of all the algorithms, and its number of iterations was only 10 when the objective function reached its optimal value. Though the SFLA algorithm shows better convergence than MDBA at the beginning, the iterations times with the optimal objective function was about twice that of MDBA. Therefore, the proposed MDBA had faster convergence speed and better identification accuracy than those of the other three algorithms in the case of small population and few iterations.

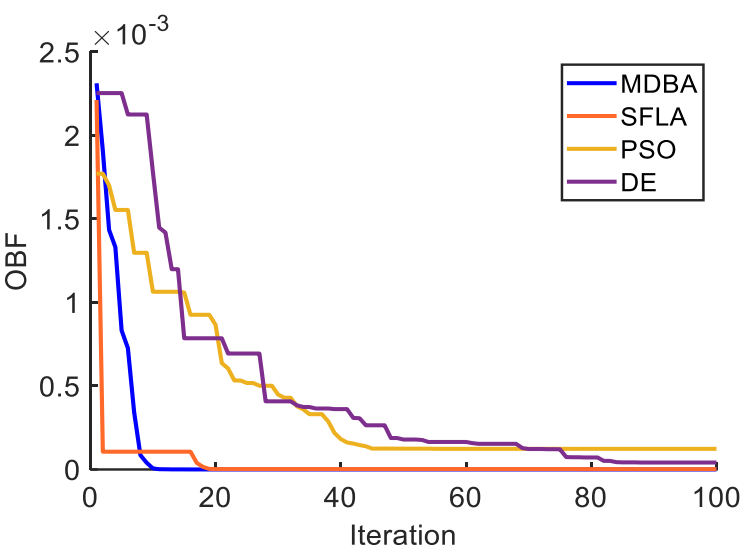

(a)

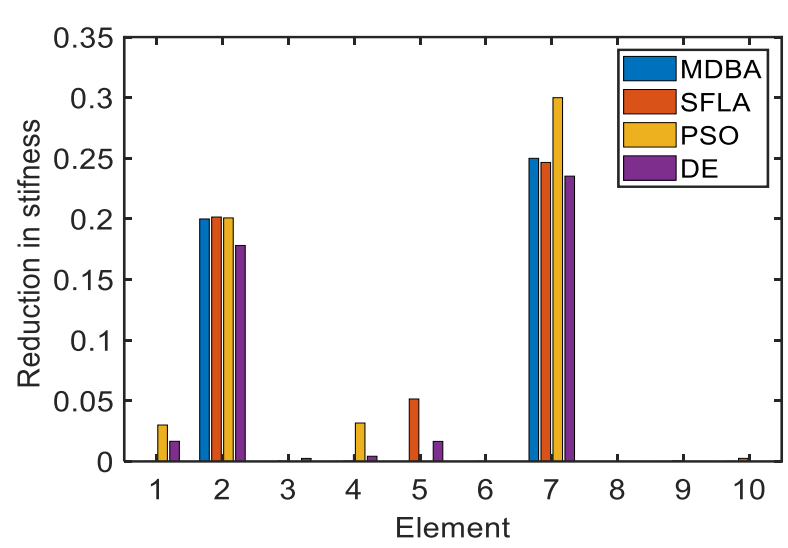

(b)

Figure 6. (a) The convergence curves achieved by four different algorithms for objection function. (b) The identification results of the damaged case by four different algorithms.

Table 1. The identification results of the damaged case by four different algorithms.

\begin{tabular}{cccc}
\hline \multirow{2}{*}{ Algorithm } & \multicolumn{2}{c}{ Damage Severity (Relative Error) } & Obvious Error Alarm \\
\cline { 2 - 4 } & Damage Element 2 & Damage Element 7 & Damage Severity @ Element \\
\hline MDBA & $19.99 \%(0.05 \%)$ & $25.00 \%(0 \%)$ & none \\
SFLA & $20.16 \%(0.80 \%)$ & $24.66 \%(1.36 \%)$ & $5.15 \% @ 5$ \\
PSO & $20.08 \%(0.40 \%)$ & $30.00 \%(20.00 \%)$ & $3.00 \% @ 1,3.17 \% @ 3$ \\
DE & $17.81 \%(10.95 \%)$ & $23.53 \%(5.88 \%)$ & none \\
\hline
\end{tabular}

In addition, when different swarm intelligence algorithms $[42,43,45]$ were employed to identify the same structure of 10-element beam, a large number of populations and iterations were required. The total number of searches by the swarm intelligence algorithm depends on population and iterations, which reflects the efficiency of the algorithm [44]. In this example, MDBA showed high efficiency under the condition of a smaller population and fewer iterations. 
To verify that the MDBA algorithm has better superiority than other improved methods of BA, MDBA was compared with other bat variants algorithms (DBA, LBA, TMBA) in this simulation. The convergence graph and identification result without noise of different bat variants are shown in Figure 7 and Table 2. It is clear in Figure 7a that under the condition of the same small population and few iterations, MDBA had a faster convergence rate than other bat variants. All of the four algorithms could accurately find the damage location of structure, but the MDBA had the best identification accuracy in damage degree, and its maximum relative error was only $0.05 \%$. The maximum relative error of other bat variants algorithms were $5.7 \%$ (DBA), $8.6 \%$ (TMBA) and $23.4 \%$ (LBA), respectively. DBA had a small error alarm in element 4 . Due to the small population and few iterations, the other three variations of BA did not perform well in the accuracy of structural damage identification. Therefore, the proposed MDBA has better superiority under the condition of a small population and few iterations.

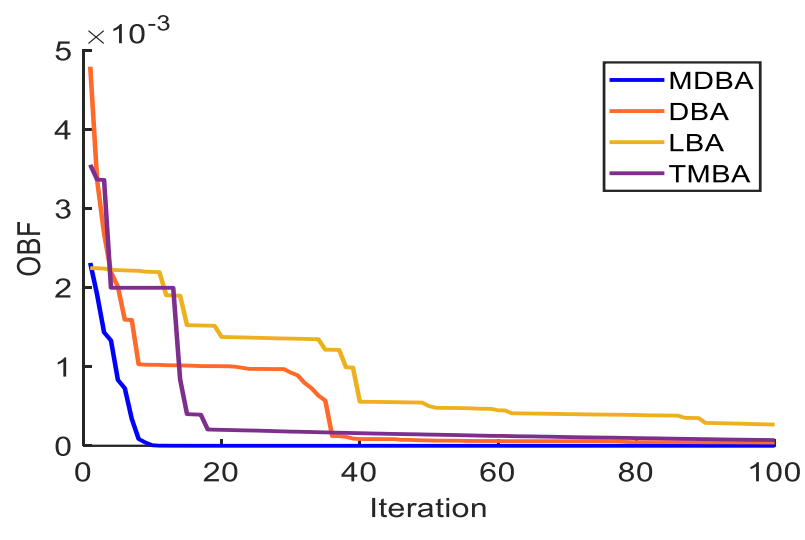

(a)

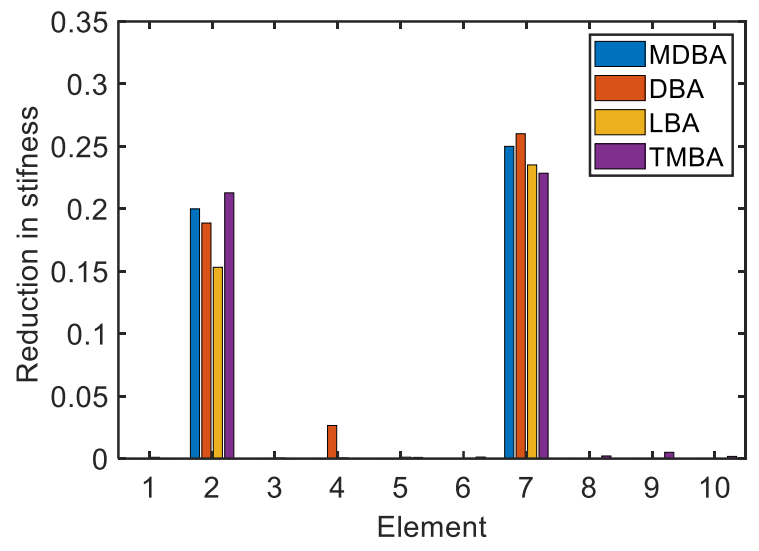

(b)

Figure 7. (a) The convergence curves achieved by other variations of BA for the objection function. (b) The identification results of the damaged case by other variations of BA.

Table 2. The identification results of the damaged case by other variations of BA.

\begin{tabular}{cccc}
\hline \multirow{2}{*}{ Algorithm } & \multicolumn{2}{c}{ Damage Severity (Relative Error) } & Obvious Error Alarm \\
\cline { 2 - 4 } & Damage Element 2 & Damage Element 7 & Damage Severity @ Element \\
\hline MDBA & $19.99 \%(0.05 \%)$ & $25.00 \%(0 \%)$ & none \\
DBA & $18.86 \%(5.70 \%)$ & $26.01 \%(4.04 \%)$ & $2.65 \% @ 4$ \\
LBA & $15.32 \%(23.40 \%)$ & $23.51 \%(5.96 \%)$ & none \\
TMBA & $21.28 \%(6.40 \%)$ & $22.85 \%(8.60 \%)$ & none \\
\hline
\end{tabular}

Next, the robustness of MDBA was verified, and different degrees of noise were applied to the modal information. The identification result under the same small population and few iterations is shown in Figure 8 and Table 3. It is clear that with the increase of noise level, the relative error of the identification results also gradually increased. However, the maximum relative error was only $5.64 \%$ in the noise levels of $5 \%$. Under the condition of a small population and few iterations, MDBA also showed good identification results and robustness in the case of different noises.

Table 3. The identification results of the damaged case by MDBA in different noise levels.

\begin{tabular}{cccc}
\hline \multirow{2}{*}{ Noise Level } & \multicolumn{2}{c}{ Damage Severity (Relative Error) } & \multirow{2}{*}{ Error Alarm Element } \\
\cline { 2 - 3 } & Damage Element 2 & Damage Element 7 & \\
\hline none & $19.99 \%(0.05 \%)$ & $25.00 \%(0 \%)$ & none \\
$1 \%$ & $19.76 \%(1.2 \%)$ & $24.43 \%(2.28 \%)$ & none \\
$5 \%$ & $20.41 \%(2.05 \%)$ & $23.59 \%(5.64 \%)$ & 10 \\
\hline
\end{tabular}




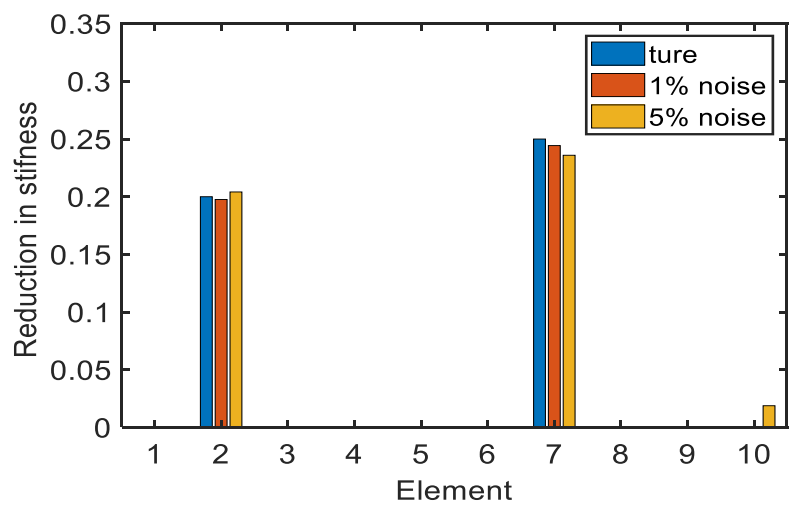

Figure 8. The identification results of the damaged case by MDBA under different noise conditions.

\subsection{Truss-Type Bridge Model}

To further verify the performance of MDBA in structural damage identification, a trusstype bridge model was used. The young's modulus of elasticity was $E=2.1 \times 10^{11} \mathrm{~Pa}$, the mass density was $\rho=7800 \mathrm{kgm}^{-3}$ and the cross-sectional area was $A=7.854 \times 10^{-5} \mathrm{~m}^{2}$, as shown in Figure 9. The first five natural frequencies and the corresponding modes of vertical degrees of freedom were used to identify the structural damage.

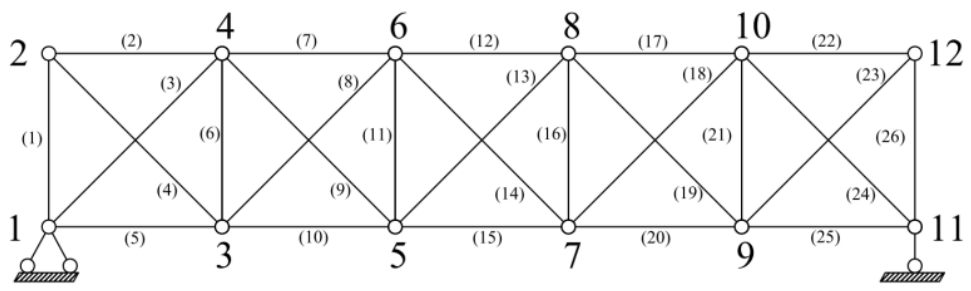

Figure 9. Truss-type bridge model.

Two kinds of damaged cases were set; case 1 was the complex damaged case with multiple damage of members; case 2 was the small damage cases with multiple damage of members, as shown in Figure 10a,b. The MDBA was verified by applying different degrees of noise to the modal information.

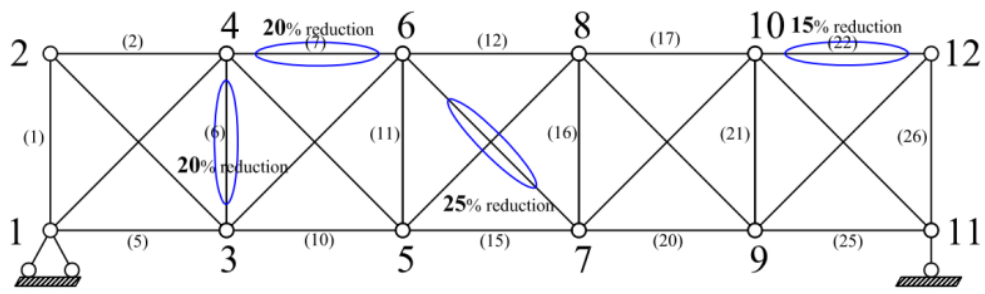

(a)

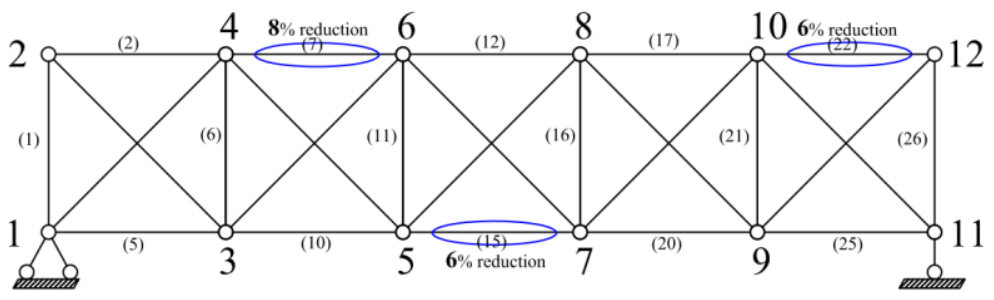

(b)

Figure 10. (a) case 1: complex damage case with multiple damage of members. (b) case 2: small damage case with multiple damage of members.

This structure was also identified by several bat variants with the same population number and iterations times in the complex multiple damage cases. The convergence 
graph and identification result diagram of the objective function of different bat variants algorithms in damage case 1 are shown in Figure 11. The detailed results of identification are shown in Table 4.

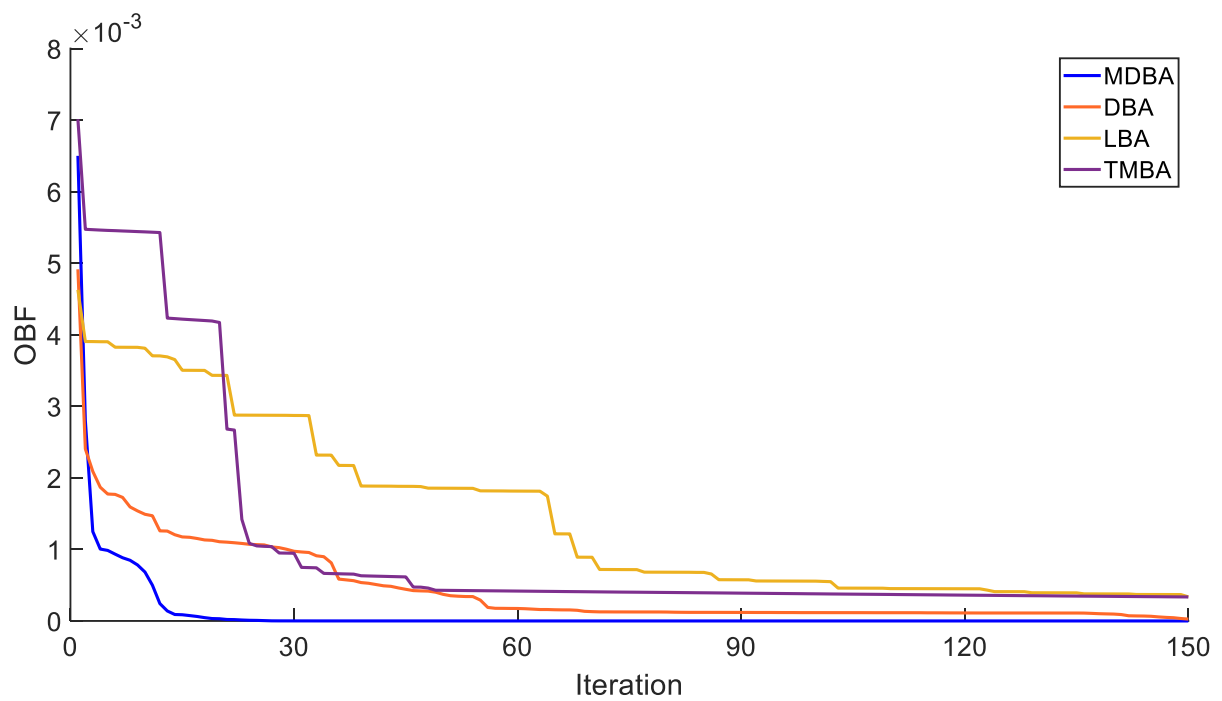

(a)

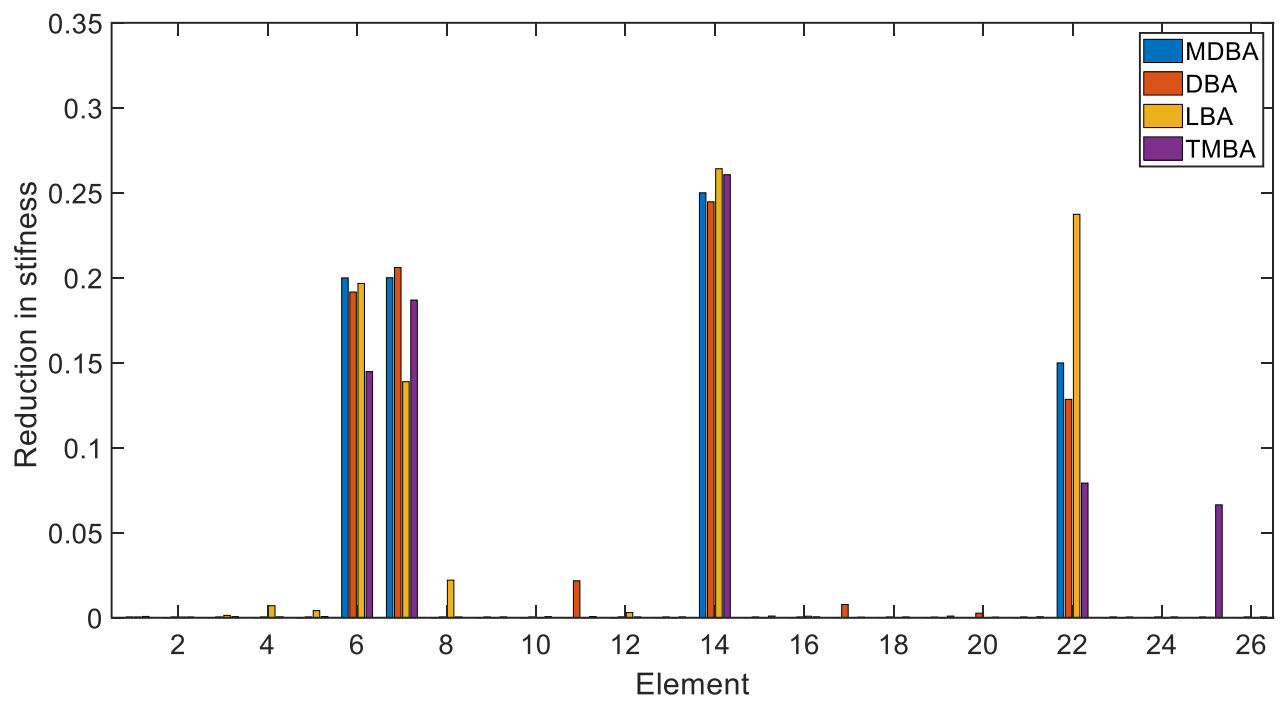

(b)

Figure 11. (a) The convergence curves achieved by other variations of BA for the objection function. (b) The identification results of the damaged case by other variations of BA.

In the complex multi-damage cases without noise, the MDBA converged to the optimal value of the function when the number of iterations reached 25 . The structural damage identification results of MDBA at damaged elements were $19.99 \%$ (element 6), $20.00 \%$ (element 7 ), $24.99 \%$ (element 14 ) and $14.99 \%$ (element 22), respectively, and the relative error was $0.07 \%-0 \%$. Under the condition of a small population and few iterations, all four algorithms could accurately find the location of structural damage, but the MDBA had the best identification accuracy in damage degree and the best and fastest convergence. Furthermore, MDBA had no error alarm where the structure was not damaged. Therefore, MDBA was superior to the other three bat variants in convergence rate and recognition accuracy. 
Table 4. The identification results of the damaged case by other variations of BA.

\begin{tabular}{cccccc}
\hline \multirow{2}{*}{ Algorithm } & \multicolumn{3}{c}{ Damage Severity (Relative Error) } & Obvious Error Alarm \\
\cline { 2 - 6 } & Damage Element 6 & Damage Element 7 & Damage Element 14 & Damage Element 22 & Damage Severity@Element \\
\hline MDBA & $19.99 \%(0.05 \%)$ & $20.00 \%(0 \%)$ & $24.99 \%(0.04 \%)$ & $14.99 \%(0.07 \%)$ & none \\
DBA & $19.16 \%(4.20 \%)$ & $20.60 \%(3.0 \%)$ & $24.47 \%(2.12 \%)$ & $12.85 \%(14.33 \%)$ & $2.17 \% @ 11$ \\
LBA & $19.67 \%(1.65 \%)$ & $13.88 \%(30.60 \%)$ & $26.41 \%(5.64 \%)$ & $23.73 \%(58.20 \%)$ & $2.21 \% @ 8$ \\
TMBA & $14.48 \%(27.60 \%)$ & $18.69 \%(6.55 \%)$ & $26.06 \%(4.24 \%)$ & $7.92 \%(47.20 \%)$ & $6.64 \% @ 25$ \\
\hline
\end{tabular}

To verify the anti-noise performance of the MDBA, the identification of two damage cases by MDBA at different noise levels were studied. The detailed results of identification are shown in Figure 12 and Table 5. It is clear that the relative error of the identification results also gradually increased with the increase of noise level, and there are two tiny misjudgments in element 11 and element 13 in case 2 . In the two damage cases, the maximum relative error was only $6.53 \%$. Especially in cases of minor damage in case 2, the maximum relative error was only $5.5 \%$. Therefore, under the condition of a small population and few iterations, the MDBA not only showed accurate identification results for complex and multiple damage cases at different noise levels, but had a high sensitivity for a minor damage case and could accurately locate and quantify the structural damage.

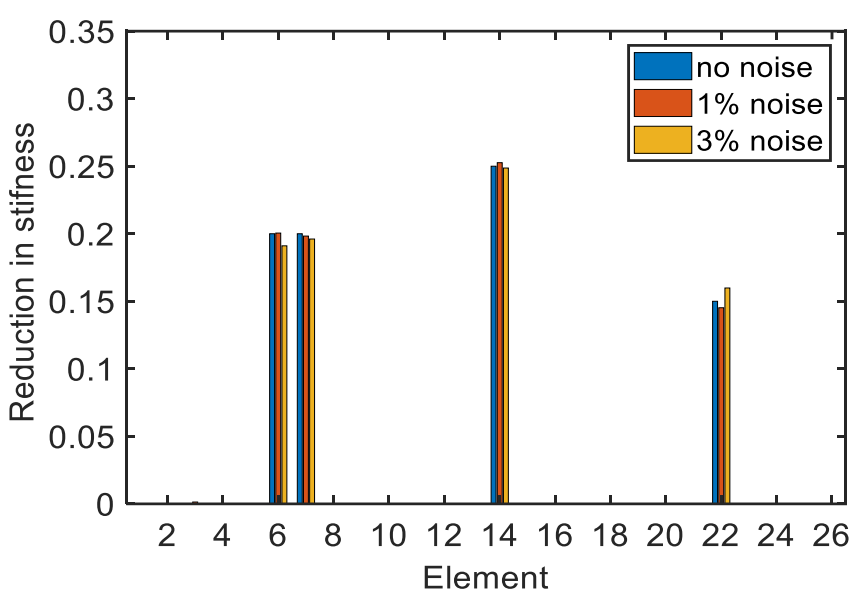

(a)

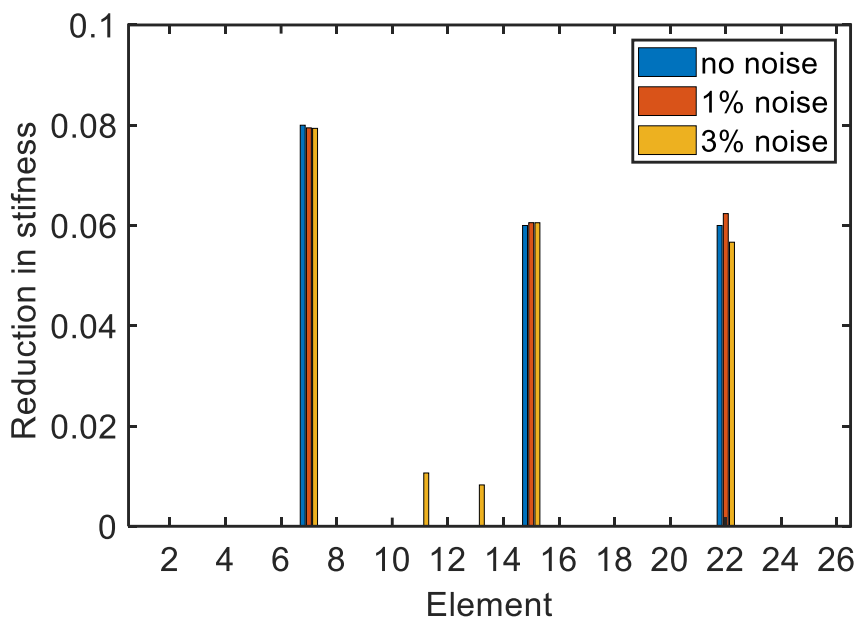

(b)

Figure 12. (a) The identification results of complex damage cases. (b) The identification results of small damage cases.

Table 5. The identification results of two damage cases by MDBA in different noise levels.

\begin{tabular}{|c|c|c|c|c|c|c|}
\hline \multirow[b]{2}{*}{ Damage Case } & \multirow[b]{2}{*}{ Noise Level } & \multicolumn{4}{|c|}{ Damage Severity (Relative Error) } & \multirow{2}{*}{$\begin{array}{c}\text { Error Alarm } \\
\text { Element }\end{array}$} \\
\hline & & $\begin{array}{c}\text { Damage } \\
\text { Element } 6\end{array}$ & $\begin{array}{c}\text { Damage } \\
\text { Element } 7\end{array}$ & $\begin{array}{c}\text { Damage } \\
\text { Element } 14\end{array}$ & $\begin{array}{c}\text { Damage } \\
\text { Element } 22\end{array}$ & \\
\hline \multirow{3}{*}{ Case 1} & None & $19.99 \%(0.05 \%)$ & $20.00 \%(0 \%)$ & $24.99 \%(0.04 \%)$ & $14.99 \%(0.07 \%)$ & none \\
\hline & $1 \%$ & $20.05 \%(0.25 \%)$ & $19.83 \%(0.85 \%)$ & $25.26 \%(1.04 \%)$ & $14.52 \%(3.20 \%)$ & none \\
\hline & $3 \%$ & $19.11 \%(4.45 \%)$ & $19.61 \%(1.95 \%)$ & $24.87 \%(0.52 \%)$ & $15.98 \%(6.53 \%)$ & none \\
\hline Damage Case & Noise Level & $\begin{array}{c}\text { Damage } \\
\text { Element } 7\end{array}$ & \multicolumn{2}{|c|}{ Damage Element 15} & $\begin{array}{c}\text { Damage } \\
\text { Element } 22\end{array}$ & $\begin{array}{c}\text { Error Alarm } \\
\text { Element }\end{array}$ \\
\hline \multirow{3}{*}{ Case 2} & None & $7.99 \%(0.13 \%)$ & \multicolumn{2}{|c|}{$6.00 \%(0 \%)$} & $5.99 \%(0.17 \%)$ & none \\
\hline & $1 \%$ & $7.95 \%(0.63 \%)$ & \multicolumn{2}{|c|}{$6.06 \%(1 \%)$} & $6.24 \%(4 \%)$ & none \\
\hline & $3 \%$ & $7.94 \%(0.75 \%)$ & \multicolumn{2}{|c|}{$6.05 \%(0.83 \%)$} & $5.67 \%(5.5 \%)$ & 11,13 \\
\hline
\end{tabular}




\section{Conclusions}

In this paper, a modified directional bat algorithm (MDBA) has been proposed for structural damage identification. By combining the individual optimal solution and elimination strategy, the optimization ability of the directional bat algorithm was strengthened. In this method, the problem of damage identification was transformed into a mathematical constrained optimization problem, and the objective function was established by frequency and mode shape. The MDBA was used to solve the constrained optimization problem. The performance of the MDBA was evaluated through the numerical simulation of a beam-type bridge and a truss-type bridge, and it was compared with other algorithms (PSO, DE, SFLA) and other variations of BA (DBA, LBA, TMBA). The following conclusions could be drawn:

1. The MDBA has the advantages of less parameters, fast convergence speed and strong global search ability, which provides a more effective and potential tool for solving structural damage identification problems.

2. For a variety of structural damage cases given different degrees of environmental noise, the MDBA could accurately locate the structural damage and estimate the damage degree, which shows its good robustness.

3. Compared with PSO, DE, SFLA and several BA variant algorithms, the MDBA has better accuracy and convergence rate in the case of the same small population and few iterations.

Under a small population and few iterations, however, the MDBA showed the fast convergence and accurate identification in the two above numerical simulations, and the robustness performance and the sensitivity to small damage were verified. It should be noted that the proposed method needs to be further verified by complex large structures and more realistic 3D models, as well as through laboratory verification or actual structure verification.

Author Contributions: Conceptualization, Y.S., L.L. and Y.L.; methodology, Y.S. and Y.L.; validation, Y.S. and L.L.; investigation, Y.S.; data curation, Y.S.; writing —original draft preparation, Y.S.; writingreview and editing, L.L.; funding acquisition, L.L. All authors have read and agreed to the published version of the manuscript.

Funding: This research was funded by the National Key R\&D Program of China via Grant No. 2017YFC1500603.

Institutional Review Board Statement: Not applicable.

Informed Consent Statement: Not applicable.

Data Availability Statement: Not applicable.

Conflicts of Interest: The authors declare no conflict of interest.

\section{References}

1. Goyal, D.; Pabla, B.S. The Vibration Monitoring Methods and Signal Processing Techniques for Structural Health Monitoring: A Review. Arch. Comput. Methods Eng. 2016, 23, 585-594. [CrossRef]

2. Gorgin, R.; Luo, Y.; Wu, Z.J. Environmental and Operational Conditions Effects on Lamb Wave Based Structural Health Monitoring Systems: A Review. Ultrasonics 2020, 105, 106114. [CrossRef]

3. Wang, J.; Yuan, J. A high-efficient multi-deme genetic algorithm with better load-balance. Int. J. Comput. Sci. Math. 2018, 9, 240-246.

4. Zhang, X.; Nguyen, H.; Bui, X.; Tran, Q.; Nguyen, D.; Bui, D.; Moayedi, H. Novel Soft Computing Model for Predicting BlastInduced Ground Vibration in Open-Pit Mines Based on Particle Swarm Optimization and XGBoost. Nat. Resour. Res. 2020, 29, 711-721. [CrossRef]

5. Pooja, P.; Chaturvedi, P.; Kumar, A.; Tomar, A. A novel differential evolution approach for constraint optimization. Int. J. Bio-Inspired Comput. 2018, 12, 254-265. [CrossRef]

6. Chen, L.; Zhou, C.; Li, X.; Dai, G. An improved differential evolution algorithm based on suboptimal solution mutation. Int. J. Comput. Sci. Math. 2017, 8, 28-34. [CrossRef] 
7. Levin, R.I.; Lieven, N.A.J. Dynamic finite element model updating using simulated annealing and genetic algorithms. Mech. Syst. Signal Process. 1998, 12, 91-120. [CrossRef]

8. Dorigo, M.; Blum, C. Ant colony optimization theory: A survey. Theor. Comput. Sci. 2005, 344, 243-278. [CrossRef]

9. Mirjalili, S.; Lewis, A. The Whale Optimization Algorithm. Adv. Eng. Softw. 2016, 95, 51-67. [CrossRef]

10. Bekdas, G.; Nigdeli, S.M.; Yang, X.S. A novel bat algorithm based optimum tuning of mass dampers for improving the seismic safety of structures. Eng. Struct. 2018, 159, 89-98. [CrossRef]

11. Mirjalili, S. The Ant Lion Optimizer. Adv. Eng. Softw. 2015, 83, 80-98. [CrossRef]

12. Eusuff, M.; Lansey, K.; Pasha, F. Shuffled frog-leaping algorithm: A memetic meta-heuristic for discrete optimization. Eng. Optim. 2006, 38, 129-154. [CrossRef]

13. Gandomi, A.; Yang, X.S.; Alavi, A. Cuckoo search algorithm: A metaheuristic approach to solve structural optimization problems. Eng. Comput. 2013, 29, 17-35. [CrossRef]

14. Mirjalili, S.; Mirjalili, S.M.; Lewis, A. Grey wolf optimizer. Adv. Eng. Softw. 2014, 69, 46-61. [CrossRef]

15. Shi, K.; Zhang, X.; Xia, S. Multiple Swarm Fruit Fly Optimization Algorithm Based Path Planning Method for Multi-UAVs. Appl. Sci. 2020, 8, 2822. [CrossRef]

16. Peng, L.; Zhu, Q.; Lv, S.; Wang, L. Effective long short-term memory with fruit fly optimization algorithm for time series forecasting. Soft Comput. 2020, 24, 15059-15079. [CrossRef]

17. Zhang, Y. Backtracking search algorithm with specular reflection learning for global optimization. Knowl. Based Syst. 2021, 212, 106546. [CrossRef]

18. Wang, L.; Peng, L.; Wang, S.; Liu, S. Advanced backtracking search optimization algorithm for a new joint replenishment problem under trade credits with grouping constraint. Appl. Soft Comput. 2020, 86, 105953. [CrossRef]

19. Huang, Y.; Shen, X.; You, X. A discrete shuffled frog-leaping algorithm based on heuristic information for traveling salesman problem. Appl. Soft Comput. 2021, 102, 107085. [CrossRef]

20. Guo, S.; Abbassi, R.; Jerbi, H.; Rezvani, A.; Suzuki, K. Efficient maximum power point tracking for a photovoltaic using hybrid shuffled frog-leaping and pattern search algorithm under changing environmental conditions. J. Clean. Prod. 2021, 297, 126573. [CrossRef]

21. Chen, C.B.; Yu, L. A hybrid ant lion optimizer with improved nelder-mead algorithm for structural damage detection by improving weighted trace lasso regularization. Adv. Struct. Eng. 2019, 23, 468-484. [CrossRef]

22. Du, D.C.; Vinh, H.H.; Trung, V.D.; Quyen, N.; Trung, N.T. Efficiency of jaya algorithm for solving the optimization-based structural damage identification problem based on a hybrid objective function. Eng. Optim. 2018, 50, 1233-1251. [CrossRef]

23. Zenzen, R.; Belaidi, I.; Khatir, S.; Wahab, M.A. A damage identification technique for beam-like and truss structures based on FRF and Bat Algorithm. Comptes Rendus Mécanique 2018, 346, 1253-1266. [CrossRef]

24. Ghiasi, R.; Fathnejat, H.; Torkzadeh, P. A three-stage damage detection method for large-scale space structures using forward substructuring approach and enhanced bat optimization algorithm. Eng. Comput. Ger. 2018, 35, 857-874. [CrossRef]

25. Huang, M.S.; Cheng, X.; Lei, Y. Structural damage identification based on substructure method and improved whale optimization algorithm. J. Civ. Struct. Health 2021, 11, 351-380. [CrossRef]

26. Ding, Z.; Zhao, Y.; Lu, Z. Simultaneous identification of structural stiffness and mass parameters based on Bare-bones Gaussian Tree Seeds Algorithm using time-domain data. Appl. Soft. Comput. 2019, 83, 105602. [CrossRef]

27. Zhu, J.; Huang, M.; Lu, Z. Bird mating optimizer for structural damage detection using a hybrid objective function. Swarm Evol. Comput. 2017, 35, 41-52. [CrossRef]

28. Yang, X.S. A new metaheuristic bat-inspired algorithm. In Nature Inspired Cooperative Strategies for Optimization (NICSO); Springer: Berlin/Heidelberg, Germany, 2010.

29. Yang, X.S.; He, X.S. Bat algorithm: Literature review and applications. Int. J. BioInspired Comput. 2013, 5, 141-149. [CrossRef]

30. He, M.; Sun, L.; Zeng, X.; Liu, W.; Tao, S. Node layout plans for urban underground logistics systems based on heuristic Bat algorithm. Comput. Commun. 2020, 154, 465-480. [CrossRef]

31. Gan, C.; Cao, W.; Wu, M.; Chen, X. A new bat algorithm based on iterative local search and stochastic inertia weight. Expert Syst. Appl. 2018, 104, 202-212. [CrossRef]

32. Liu, Q.; Li, J.; Wu, L.; Wang, F.; Xiao, W. A novel bat algorithm with double mutation operators and its application to low-velocity impact localization problem. Eng. Appl. Artif. Intel. 2020, 90, 103505. [CrossRef]

33. Cui, Z.; Zhang, C.; Zhao, Y.; Shi, Z. Adaptive Bat Algorithm Optimization Strategy for Observation Matrix. Appl. Sci. 2019, 9 , 3008. [CrossRef]

34. Al-Betar, M.; Awadallah, M. Island bat algorithm for optimization. Expert Syst. 2018, 107, 126-145. [CrossRef]

35. Chakri, A.; Khelif, R.; Benouaret, M.; Yang, X.S. New directional bat algorithm for continuous optimization problems. Expert Syst. Appl. 2017, 69, 159-175. [CrossRef]

36. Fister, I., Jr.; Fister, D.; Yang, X.S. A hybrid bat algorithm. arXiv 2013, arXiv:1303.6310.

37. Ghanem, W.; Jantan, A. An enhanced bat algorithm with mutation operator for numerical optimization problems. Neural Comput. 2017, 1-35. [CrossRef]

38. Hong, W.; Li, M.; Geng, J.; Zhang, Y. Novel chaotic bat algorithm for forecasting complex motion of floating platforms. Appl. Math. Model. 2019, 72, 425-443. [CrossRef]

39. Jordehi, A.R. Chaotic bat swarm optimisation (CBSO). Appl. Soft Comput. 2015, 26, 523-530. [CrossRef] 
40. Ghannadi, P.; Kourehli, S. Structural damage detection based on MAC flexibility and frequency using moth-flame algorithm. Struct. Eng. Mech. 2019, 70, 649-659.

41. Fallah, N.; Vaez, S.; Mohammadzadeh, A. Multi-damage identification of large-scale truss structures using a two-step approach. J. Build. Eng. 2018, 19, 494-505. [CrossRef]

42. Shabbir, F.; Khan, M.; Ahmad, N.; Tahir, M.; Ejaz, N.; Hussain, J. Structural Damage Detection with Different Objective Functions in Noisy Conditions Using an Evolutionary Algorithm. Appl. Sci. 2017, 7, 1245. [CrossRef]

43. Chen, C.; Pan, C.; Chen, Z.; Yu, L. Structural damage detection via combining weighted strategy with trace Lasso. Adv. Struct. Eng. 2019, 22, 597-612. [CrossRef]

44. Rauf, H.; Malik, S.; Shoaib, U.; Irfan, M.; Lali, M. Adaptive inertia weight Bat algorithm with Sugeno-Function fuzzy search. Appl. Soft Comput. 2020, 90, 106159. [CrossRef]

45. Georgioudakis, M.; Plevris, V. A Combined Modal Correlation Criterion for Structural Damage Identification with Noisy Modal Data. Adv. Civ. Eng. 2018, 2018, 3183067. [CrossRef] 